\title{
Broad Repetition-Rate Tunable Quantum-Dot External-Cavity Passively Mode-Locked Laser with Extremely Narrow RF Linewidth
}

\author{
Ying Ding, ${ }^{1, *}$ Maria Ana Cataluna, ${ }^{1}$ Daniil Nikitichev, ${ }^{1}$ Igor Krestnikov, ${ }^{2}$ Daniil Livshits, ${ }^{2}$ \\ and Edik Rafailov ${ }^{1}$ \\ ${ }^{1}$ School of Engineering, Physics and Mathematics, The University of Dundee, Dundee, DDI 4HN, UK \\ ${ }^{2}$ Innolume GmbH, Konrad-Adenauer-Allee 11, 44263 Dortmund, Germany
}

\begin{abstract}
We report on a systematic investigation of a repetition-rate-tunable quantum-dot external-cavity passively mode-locked laser with a quasi-continuous frequency tuning range from $1 \mathrm{GHz}$ to a record-low value of $191 \mathrm{MHz}$. A nearly constant pulse peak power at the different pulse repetition rates is revealed in the continuous frequency tuning range. The trend and optimization of the stable fundamental mode-locking are presented and interpreted. RF linewidth of a record value of $\sim 30 \mathrm{~Hz}$ is demonstrated, which indicates the low noise operation and high stability of the quantum-dot external-cavity passively mode-locked laser.
\end{abstract}

\footnotetext{
*E-mail address: y.ding@ dundee.ac.uk
} 
Semiconductor lasers with broad tunability of the pulse repetition rate in the range of $\mathrm{MHz}$ to $\mathrm{GHz}$ are very useful for non-linear imaging techniques, especially in the bio-medical field. ${ }^{1,2)}$ Quantum-dot external-cavity passively mode-locked lasers (QD-ECMLLs) are excellent candidates for this kind of applications due to QD lasers inherent merits of low threshold current density, low optical losses and low noise characteristics ${ }^{3,4)}$ as well as the versatility that external-cavity configurations can offer for broad tunability of the pulse repetition rate.

In our previous work, we demonstrated a QD-ECMLL incorporating a QD semiconductor saturable absorber mirror (SESAM), ${ }^{5)}$ generating pulses at repetition rates ranging from $1.5 \mathrm{GHz}$ to $350 \mathrm{MHz}$. In a more recent investigation, ${ }^{6}$ a QD-ECMLL is demonstrated with pulse repetition rate as low as $310 \mathrm{MHz}$. We have demonstrated very recently the generation of high-peak-power picosecond pulses with repetition frequency from 2.4 $\mathrm{GHz}$ to $1.14 \mathrm{GHz}^{7)}$ using an output coupler of $96 \%$ transmissivity as the external cavity facet. On the other hand, QD monolithic passively mode-locked lasers (MLLs) have shown very low noise performance, with the demonstration of record-low RF linewidth of $500 \mathrm{~Hz}$ at gigahertz repetition rates. ${ }^{8)}$ A quantum-dash-based passively MLL at $17 \mathrm{GHz}$ with a RF linewidth narrowing down to $500 \mathrm{~Hz}$ by using external optical feedback was also reported. ${ }^{9)}$ Lately, with the same approach of external optical feedback, a RF linewidth of $350 \mathrm{~Hz}$ was demonstrated by QD passively MLL with a repetition rate of $5 \mathrm{GHz},{ }^{10)}$ representing the narrowest linewidth reported for QD passive MLLs in spite of whether a linewidth narrowing technique is used. Very recently, we achieved a RF linewidth of $\sim 300 \mathrm{~Hz}$ by a QD-ECMLL ${ }^{11)}$ at a repetition frequency of $323 \mathrm{MHz}$.

In this paper we present our latest results achieved with a QD-ECMLL $(\lambda=1.27 \mu \mathrm{m})$ based on a two-section superluminescent diode chip and a 53\% output coupler as an external cavity facet. A broad continuous frequency tuning range from $1 \mathrm{GHz}$ to $191 \mathrm{MHz}$ and $\mathrm{RF}$ linewidth of $\sim 30 \mathrm{~Hz}$ are demonstrated. To the best of our knowledge, the pulse repetition rate 
of $191 \mathrm{MHz}$ is the lowest frequency ever to be demonstrated for a QD-ECMLL, and -3-dB RF linewidth of $\sim 30 \mathrm{~Hz}$ is the narrowest value observed to date for any passively modelocked semiconductor laser. The trends of mode-locking performance are also illustrated and discussed.

The QD chip structure investigated here was grown on an $n^{+}$-GaAs (100) substrate by molecular beam expitaxy (MBE) which contained 10 layers of self-assembled InAs/GaAs quantum dots. The waveguide in the gain section is bent and terminated at an angle of $7^{\circ}$ relative to the cleaved facet, in combination with an antireflection (AR) coating $\left(\mathrm{R} \sim 10^{-5}\right)$, while the back facet was high-reflection (HR) coated (R 95\%). The total chip length is $4 \mathrm{~mm}$, with a $600-\mu \mathrm{m}$-long saturable absorber section placed near the back facet. A simplified schematic of the QD-ECMLL is depicted in Fig.1. The chip was kept at $20^{\circ} \mathrm{C}$ using thermoelectric temperature control during the measurement. The RF spectra with a $10-\mathrm{GHz}$ span at the mode-locking operation conditions of reverse bias of $8 \mathrm{~V}$ and forward current of $300 \mathrm{~mA}$ were recorded for the variable fundamental mode-locking repetition rate from $1 \mathrm{GHz}$ to $191 \mathrm{MHz}$ as shown in Fig. 2. The corresponding total optical cavity length varies from 15 $\mathrm{cm}$ to $78.5 \mathrm{~cm}$. The continuously tunable range and accuracy of the pulse repetition rate depends on the adjustment limitation and minimum achievable incremental movement of translation stage. As such, the possible control precision of the frequency can be up to $700 \mathrm{~Hz}$ and $30 \mathrm{~Hz}$ at the repetition rate of $1 \mathrm{GHz}$ and $200 \mathrm{MHz}$, respectively, for the translation stage with a $0.1 \mu \mathrm{m}$ step motion. The large number of harmonics in the RF spectra indicates the high quality of mode-locking for all cases, as shown in Fig. 2.

Further investigations of the mode-locking performance were carried out for the different repetition rates. The region of stable fundamental mode-locking operation was plotted for the typical repetition rate of $750 \mathrm{MHz}$ as shown in Fig. 3. As mentioned in Ref. 7, the external cavity optical feedback from the output coupler should be optimized for each 
operating point in order to achieve stable fundamental mode-locking. In the robust operation area highlighted in Fig. 3, the stable fundamental mode-locking can be obtained more easily. In this case, the setup has a high anti-jamming ability, e.g. resilience to vibration which is indicative of great application prospect. The pulse duration of less than 7 ps can be obtained under the reverse bias of $8 \mathrm{~V}$ and forward current of $300 \mathrm{~mA}$.

From Fig. 3, it can be observed that for increasing values of current applied to the gain section, the region of robust fundamental mode-locking became narrower, as the laser displayed an increasing tendency for operating in a harmonic mode-locking regime, rather than in stable fundamental mode-locking, which is a well-known phenomenon also observed in monolithic QD MLLs. ${ }^{12)}$ For the other pulse repetition rates, similar mode-locking operation trends can be observed.

The peak power remains nearly constant under certain operation conditions (especially at a low gain current and a high reverse bias, e. g. $\sim 0.5 \mathrm{~W}$ at $-8 \mathrm{~V}, 300 \mathrm{~mA}$,) with different pulse repetition rates. The average power was found to be proportional to the repetition rate, which implies that the constant pulse energy required to saturate the absorber is independent of repetition rate under certain operation conditions. This result is in principle in keeping with the conclusion reported in Ref. 6, where there is mention of constant pulse energy irrespective of pulse duration, repetition rate, reverse bias or drive current, for optimum mode-locking conditions. However, the fact of increasing average power and somewhat increased peak power with the increasing gain current for a certain reverse bias at a certain repetition rate (e.g. as represented in Fig. 4) reveal an important issue for the employed external cavity setup (Fig. 1) in this study.

For sustaining a stable fundamental mode-locking, the net gain should be low enough in a wide time window for the relatively long pulse roundtrip time in the long cavity so as to suppress the high-order harmonic mode-locking. The above-mentioned optimizing of the 
optical feedback is to decrease the feedback appropriately, thereby decreasing the net gain. As a guidance to this optimization procedure, it was noted that the best conditions for fundamental mode-locking corresponded to a certain value of photocurrent in the absorber which should be kept at 6-8 $\mathrm{mA}$ during the feedback optimization, independently of how much current was applied to the gain section - this corresponds to the optimal conditions of absorption saturation under a certain reverse bias, in order to achieve fundamental modelocking operation. On the contrary, the photocurrent may be up to a few tens of mA with the maximized optical feedback, which definitely corresponds to an unstable fundamental modelocking. Consequently, the average output power (pulse energy) of stable fundamental modelocking can be increased with the increasing gain current due to the fixed transmissivity of the output coupler and the adjustable optical feedback. Obviously, for a higher gain current, a lower optical feedback is required to keep the balance of gain and loss to achieve a stable fundamental mode-locking. Accordingly, it can be predicted that output coupler with higher transmissivity could produce a higher output power, but the resonance formation and optical feedback adjustment will be challenging at a low frequency operation, and the threshold current will be higher because of higher cavity loss.

We have also explored the RF linewidth for the different repetition rates. QD modelocked lasers have been regarded as low-noise sources due to enabling low threshold current densities and reduced values of amplified spontaneous emission (ASE). ${ }^{13)}$ Indeed, spontaneous fluctuations represent one of the major sources of timing jitter, as it imparts random fluctuations in the index of refraction, thereby affecting the round-trip time and thus the timing of the pulses. In the case of ECMLL, mechanical instability can also contribute to timing jitter. An improvement in the phase noise and timing jitter is expected in a stable external-cavity laser when comparing to its monolithic counterpart, because the active waveguide occupies only a fraction of the optical cavity. ${ }^{14)}$ 
After minimizing the mechanical instabilities of the setup as much as possible, extremely narrow RF linewidth was observed within the tuning range as expected, especially for the low frequencies. For a sample of randomly selected repetition rates, such as $257 \mathrm{MHz}$, $238 \mathrm{MHz}$ and $191 \mathrm{MHz}$, narrow linewidth lower than $100 \mathrm{~Hz}$ can be attained easily. Here, a Lorentzian lineshape fitting is employed to determine the $-3 \mathrm{~dB}$ RF linewidth from our measurements. Higher measurement resolution is also employed for this narrow linewidth investigation. With the optimized setup adjustment and operation conditions, a record narrow $\mathrm{RF}$ linewidth of $\sim 30 \mathrm{~Hz}$ was achieved from the fundamental mode-locking under $8 \mathrm{~V}$ reverse bias and $300 \mathrm{~mA}$ forward current with a pulse repetition rate of $281 \mathrm{MHz}$, as shown in Fig. 5 . These observations indicate the very low noise and robust mechanical stability that even can be compared to the hybrid or active MLLs. This result further verified the current view that QD lasers offer major advantages for low-noise operation, for both high and low pulse repetition rates.

In summary, we have investigated experimentally the broad repetition-rate tunable QDECMLL with a frequency tuning range from $1 \mathrm{GHz}$ to a record-low value of $191 \mathrm{MHz}$. The RF linewidth of $\sim 30 \mathrm{~Hz}$ is considered as the record for passively MLLs reported up-to-date, which implies a remarkable prospect for applications.

\section{Acknowledgement}

The work was funded within the Seventh Framework Program "FAST-DOT", through Grant No. 224338. M. A. Cataluna acknowledges also financial support through a Royal Academy of Engineering/EPSRC Research Fellowship. The authors would like to acknowledge helpful discussions with Mattia Rossetti (Politecnico di Torino). 


\section{References}

1 S.-W. Chu, T.-M. Liu, C.-K. Sun, C.-Y. Lin, and H.-J. Tsai: Opt. Express 11 (2003) 933.

2 H. Yokoyama, A. Sato, H.-C. Guo, K. Sato, M. Mure, and H. Tsubokawa: Opt. Express 16 (2008) 17752.

3 H. Y. Liu, D. T. Childs, T. J. Badcock, K. M. Groom, I. R. Sellers, M. Hopkinson, R. A. Hogg, D. J. Robbins, D. J. Mowbray, and M. S. Skolnick: IEEE Photonics Technol. Lett. 17 (2005) 1139.

4 M. G. Thompson, A. Rae, M. Xia, R. V. Penty, and I. H. White: IEEE J. Sel. Top. Quantum Electron. 15 (2009) 661.

5 A. D. McRobbie, M. A. Cataluna, S. A. Zolotovskaya, D. A. Livshits, W. Sibbett, and E. U. Rafailov: Electron. Lett. 43 (2007) 812.

6 M. Xia, M. G. Thompson, R. V. Penty, and I. H. White: 2008 Conference on Lasers and Electro-Optics \& Quantum Electronics and Laser Science Conference (CLEO/QELS), 2008, 1-9, p. 1137.

7 Y. Ding, D. I. Nikitichev, I. Krestnikov, D. Livshits, M. A. Cataluna, and E. U. Rafailov: Electron. Lett. 46 (2010) 1516 [Errata 46 (2010) 1702].

8 G. Carpintero, M. G. Thompson, R. V. Penty, and I. H. White: IEEE Photonics Technol. Lett. 21 (2009) 389.

9 K. Merghem, R. Rosales, S. Azouigui, A. Akrout, A. Martinez, F. Lelarge, G.-H. Duan, G. Aubin, and A. Ramdane: Appl. Phys. Lett. 95 (2009)131111.

10 C.-Y. Lin, F. Grillot, N. A. Naderi, Y. Li, and L. F. Lester: Appl. Phys. Lett. 96 (2010) 051118.

11 M. A. Cataluna, Y. Ding, D. Nikitichev, K. A. Fedorova, and E. U. Rafailov: to be published in IEEE J. Sel. Top. Quantum Electron. (2011). 
12 E. A. Viktorov, P. Mandel, M. Kuntz, G. Fiol, D. Bimberg, A. G. Vladimirov, and M. Wolfrum: Appl. Phys. Lett. 91 (2007) 231116.

13 T. W. Berg and J. Mork: Appl. Phys. Lett. 82 (2003) 3083.

14 P. Vasil'ev: Ultrafast Diode Lasers: Fundamentals and Applications (Boston: Artech House, 1995) Chap. 4 p. 140. 


\section{Figure captions}

Fig. 1. Configuration of a QD-ECMLL and the experimental setup. OC: output coupler $(\mathrm{R}=47 \%, \mathrm{~T}=53 \%)$; $\mathrm{TS}$ : motorized translation stage; OI: optical isolator; HWP: half wave plate; SMF: single-mode fiber; FS: fiber splitter; OSA: optical spectrum analyzer; Autoco: autocorrelator; Osc: oscilloscope; PD: photo detector; RFSA: RF spectrum analyzer.

Fig. 2. Representative RF spectra with $10 \mathrm{GHz}$ span at the reverse bias of $8 \mathrm{~V}$ and forward current of $300 \mathrm{~mA}$ recorded for the variable repetition rate from $1 \mathrm{GHz}$ to $191 \mathrm{MHz}$.

Fig. 3. Regime of stable fundamental mode-locking with repetition rate of $750 \mathrm{MHz}$.

Fig. 4. Peak power and average power against forward current with $8 \mathrm{~V}$ reverse bias at the repetition rate of $375 \mathrm{MHz}$.

Fig. 5. RF spectrum measured under $8 \mathrm{~V}$ reverse bias and $300 \mathrm{~mA}$ forward current, at a 281 $\mathrm{MHz}$ pulse repetition rate. Inset: $\mathrm{RF}$ spectrum with a $10 \mathrm{KHz}$ span, with a $-3 \mathrm{~dB}$ linewidth of $\sim 30 \mathrm{~Hz}$ (obtained after Lorentzian fit). The resolution and video bandwidth for this acquisition were $30 \mathrm{~Hz}$ and $3 \mathrm{~Hz}$, respectively. 


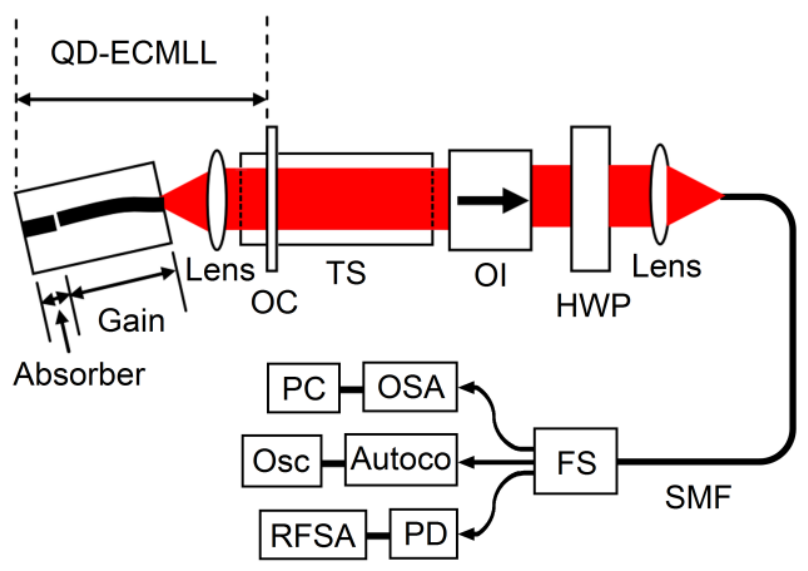

Fig. 1 


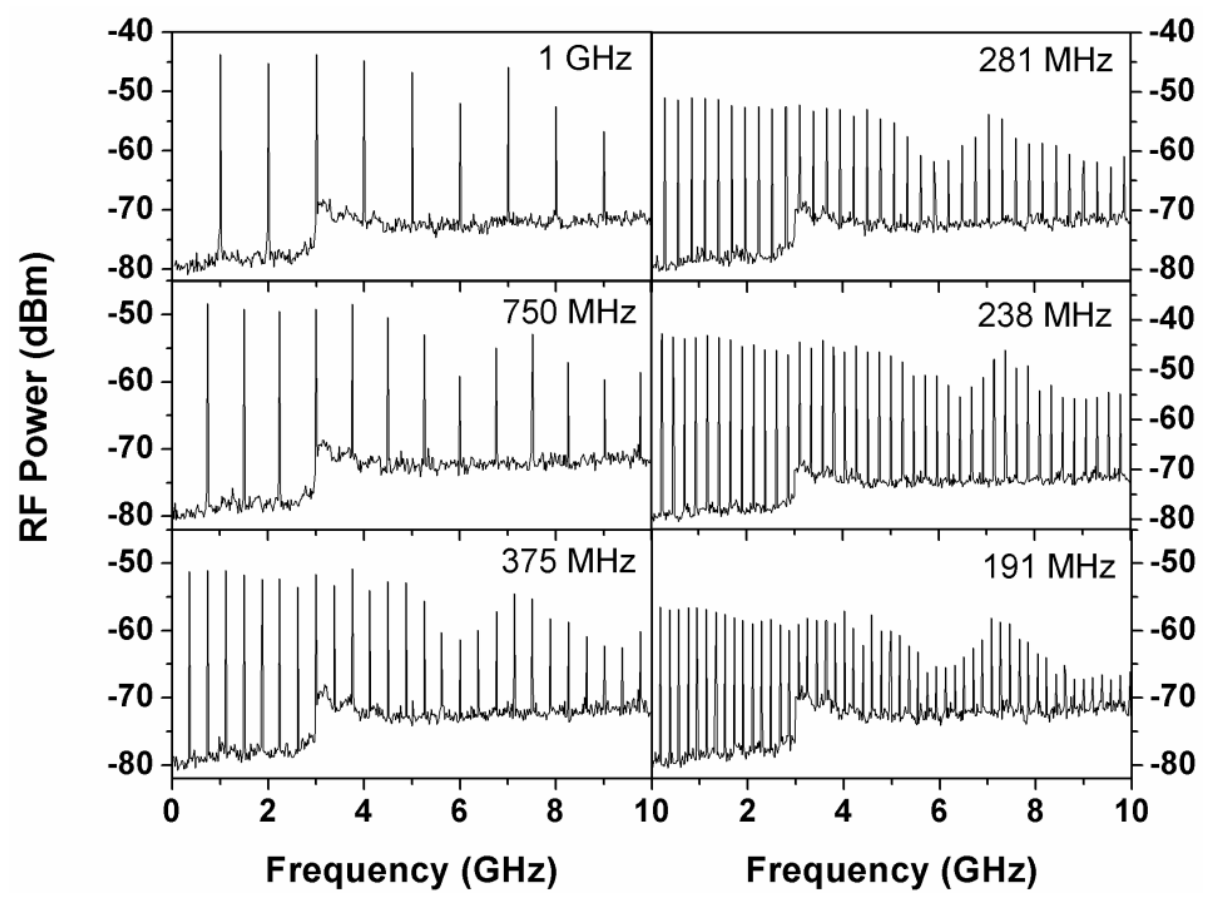

Fig. 2 


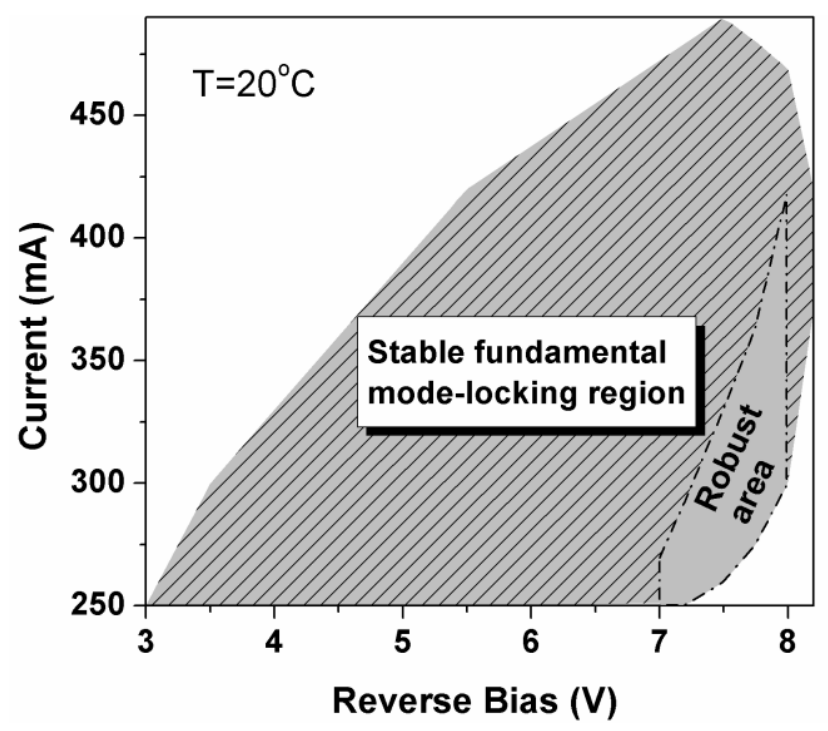

Fig. 3 


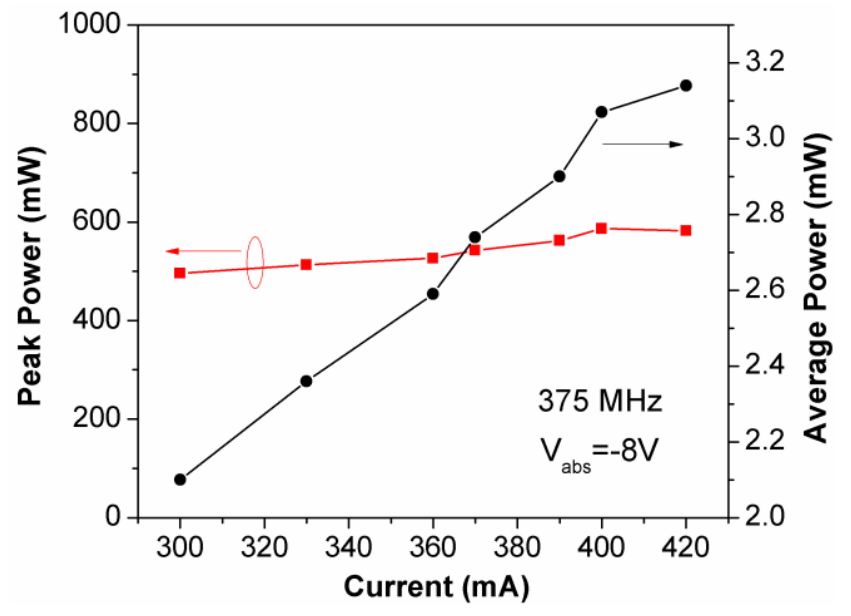

Fig. 4 


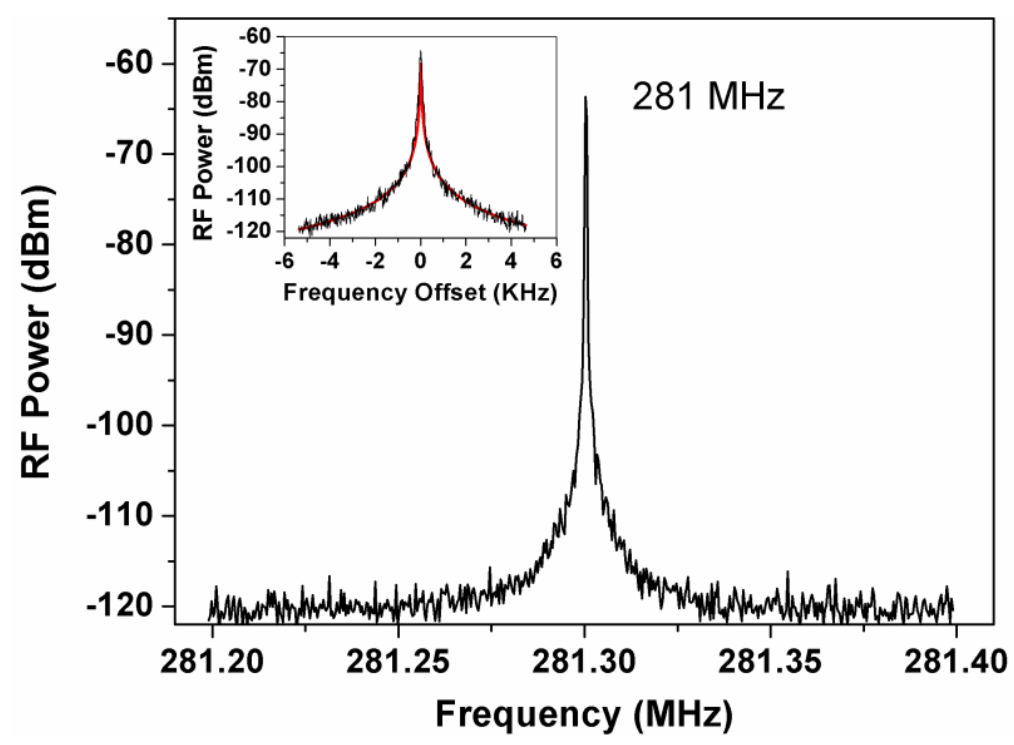

Fig. 5 